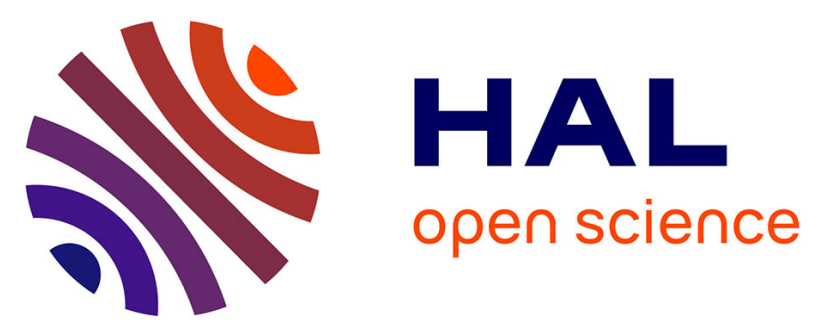

\title{
Oxidative stress, mitochondrial permeability transition pore opening and cell death during hypoxia-reoxygenation in adult cardiomyocytes.
}

Rana Assaly, Alexandra d'Anglemont de Tassigny, Stéphanie Paradis, Sophie Jacquin, Alain Berdeaux, Didier Morin

\section{To cite this version:}

Rana Assaly, Alexandra d'Anglemont de Tassigny, Stéphanie Paradis, Sophie Jacquin, Alain Berdeaux, et al.. Oxidative stress, mitochondrial permeability transition pore opening and cell death during hypoxia-reoxygenation in adult cardiomyocytes.. European Journal of Pharmacology, 2012, 675 (1-3), pp.6-14. 10.1016/j.ejphar.2011.11.036 . inserm-00680277

\section{HAL Id: inserm-00680277 https://www.hal.inserm.fr/inserm-00680277}

Submitted on 19 Mar 2012

HAL is a multi-disciplinary open access archive for the deposit and dissemination of scientific research documents, whether they are published or not. The documents may come from teaching and research institutions in France or abroad, or from public or private research centers.
L'archive ouverte pluridisciplinaire HAL, est destinée au dépôt et à la diffusion de documents scientifiques de niveau recherche, publiés ou non, émanant des établissements d'enseignement et de recherche français ou étrangers, des laboratoires publics ou privés. 
Oxidative stress, mitochondrial permeability transition pore opening and cell death during hypoxia-reoxygenation in adult cardiomyocytes.

Rana Assaly; Alexandra d'Anglemont de Tassigny; Stéphanie Paradis; Sophie Jacquin; Alain Berdeaux; Didier Morin.

INSERM U955 équipe 03, F-94010, Créteil, France ; Université Paris-Est Créteil, F-94010, Créteil, France.

Address for correspondence:

Didier Morin, phD

Faculté de Médecine de Créteil

INSERM U955 équipe 03

8, rue du Général Sarrail

94010 Créteil Cedex, France

Téléphone : +33 (0) 148813661

Fax : +33 (0) 148813661

e-mail : didier.morin@inserm.fr 


\section{Abstract:}

Reactive oxygen species production is necessary to induce cell death following hypoxia/reoxygenation but the effect of reactive oxygen species produced during hypoxia on mitochondrial permeability transition pore (mPTP) opening and cell death is not established. Here we designed a model of hypoxia/reoxygenation in isolated cardiomyocytes measuring simultaneously reactive oxygen species production, mPTP opening and cell death in order (i) to establish a causal relationship between them, (ii) to investigate the roles of various reactive oxygen species in MPTP opening. The percentage of cardiomyocytes exhibiting mPTP opening during reoxygenation increased with the duration of hypoxia. Antioxidants increased the time to MPTP opening when present during hypoxia but not at reoxygenation. This was associated with a drop in hydroxyl radical and hydrogen peroxide during hypoxia and the first minutes of reoxygenation. The increase in time to mPTP opening was accompanied by an improvement in cell viability reflected by maintenance of superoxide production at reoxygenation. Cyclosporin A delayed both the time to MPTP opening and cell death despite maintenance of reactive oxygen species production during hypoxia. These findings demonstrate that reactive oxygen species production precedes MPTP opening and that reactive oxygen species produced during hypoxia, particularly hydroxyl radicals and hydrogen peroxide, are necessary to induce MPTP opening which depends on hypoxia duration.

Keywords: adult cardiomyocytes, hypoxia/reoxygenation, mitochondria, permeability transition pore, reactive oxygen species. 


\section{Introduction:}

The obligatory restoration of blood flow following an ischemic episod is well associated with the so called "reperfusion injury" responsible for tissue injury and ultimately to cell death in part related to the production of a burst in reactive oxygen species within minutes of reperfusion (Yellon and Hausenloy, 2007; Murphy and Steenbergen, 2008). However, and despite a low tension in oxygen, a significant reactive oxygen species generation during the ischemic phase was also reported and associated with reperfusion-induced cell death (Becker et al., 1999; Robin et al., 2007).

One mechanism prominent in this injury is an increase in permeability of the mitochondrial membranes due to opening of the mitochondrial permeability transition pore (mPTP) greatly enhanced by adenine nucleotide depletion, calcium influx, elevated phosphate and oxidative stress (Halestrap, 2009). Conversely, the increase in resistance to mPTP opening with ischemic or pharmacological induced pre- and post-conditionings is an obvious effective target for cardioprotection (Halestrap et al., 2004; Obame et al., 2008; Morin et al., 2009 , Ovize et al., 2010) and drugs that decrease oxidative stress usually inhibit mPTP opening (Adlam et al., 2005; Javadov et al., 2003; Townsend et al., 2007). However, and despite extensive research, the exact mechanisms linking the temporal sequence of reactive oxygen species production, membrane alterations, mPTP opening and cell death still remain partly unclear. Many authors strongly suggest that oxidative stress is the link between excessive calcium influx, inorganic phosphate accumulation and mPTP opening seen under conditions such as ischemia/reperfusion (Kowaltowski et al., 2001; Di Lisa et al., 2009) . Otherwise, it is well demonstrated that reactive oxygen species production in isolated 
cardiomyocytes during hypoxia is involved in cell death (Robin et al., 2007) but there is no information to date about the effect of this production on $\mathrm{MPTP}$ opening. We hypothesized that reactive oxygen species production during hypoxia might play a role on mPTP opening following hypoxia/reoxygenation.

Therefore, we designed an in vitro model of hypoxia/reoxygenation in freshly isolated adult rat cardiomyocytes in order (1) to evaluate sequentially and simultaneously the kinetics of reactive oxygen species generation, mPTP opening and cell death during hypoxia/reoxygenation using fluorescent dyes and (2) to investigate the roles of various types of reactive oxygen species which participate to mPTP opening and cell death following hypoxia/reoxygenation.

\section{Materials and methods:}

All animals procedures used in this study were in accordance with the European Community Council Directive (86-609/87-848 EEC) and recommendations of the French Ministère de l'Agriculture.

\subsection{Isolation of Adult cardiomyocytes.}

Adult rat ventricular cardiomyocytes were obtained from hearts of male Wistar rats (260-300g) as described previously by the Alliance for Cellular Signaling (AfCS) (Procedure protocols: PP0000012; http://www.signaling-gateway.org). Briefly, animals were anesthetized with sodium pentobarbital $(50 \mathrm{mg} / \mathrm{kg}$ IP) and heparinized and their hearts were removed into an ice-cold $\mathrm{Ca}^{2+}$ free perfusion buffer (mM: $\mathrm{NaCl} 113, \mathrm{KCl} 4.7, \mathrm{KH}_{2} \mathrm{PO}_{4} 0.6, \mathrm{Na}_{2} \mathrm{HPO}_{4}$ 0.6, $\mathrm{MgSO}_{4} 1.2, \mathrm{NaHCO}_{3}$ 12, $\mathrm{KHCO}_{3} 10$, HEPES 10, taurine 30, 2,3-butanedione monoxime 10, glucose 5.5 at $\mathrm{pH}$ 7.4). The hearts were retrogradely-perfused at constant pressure on a 
Langendorff apparatus with the perfusion buffer for $3 \mathrm{~min}$ at $37^{\circ} \mathrm{C}$ then switched to a digestion buffer containing $\mathrm{Ca}^{2+}(12.5 \mu \mathrm{M})$, liberase $\mathrm{TM}(0.1 \mathrm{mg} / \mathrm{ml}$; Roche diagnostics, Meylan, France) and trypsin $(0.14 \mathrm{mg} / \mathrm{ml}$, Sigma, Saint-Quentin Fallavier, France). After digestion, the left ventricle was minced, filtered and allowed to sediment by gravity. The cellular pellet was washed several times with a perfusion buffer containing $5 \%$ bovine calf serum (Gibco, Invitrogen corporation, Cergy-Pontoise, France) and increasing concentrations of $\mathrm{Ca}^{2+}(\mu \mathrm{M}: 62,112,212$, $500,1000)$. Cells were plated in $35 \mathrm{~mm}$ Petri dishes precoated with laminin $(10 \mu \mathrm{g} / \mathrm{ml})$ and containing M199 (Invitrogen, Cergy-Pontoise, France). Cells were allowed to attach for $2 \mathrm{~h}$ in humidified $5 \% \mathrm{CO}_{2}-95 \%$ air at $37^{\circ} \mathrm{C}$ and then washed once to remove unattached cells before being used.

\subsection{Hypoxia/reoxygenation in adult rat cardiomyocytes.}

Petri dishes containing adherent contracting cells were mounted on a heated $\left(37^{\circ} \mathrm{C}\right)$ perfusion chamber (Warner Instruments Inc, Hamden, CT). A pump was used to perfuse Tyrode's modified solution at a rate of $0.5 \mathrm{ml} / \mathrm{min}$. The chamber was connected to a gas bottle diffusing a constant stream of $\mathrm{O}_{2}(21 \%) ; \mathrm{N}_{2}(74 \%)$ and $\mathrm{CO}_{2}(5 \%)$ maintaining a partial $\mathrm{O}_{2}$ pressure at $21 \%$. $\mathrm{pO}_{2}$ within the chamber was measured continuously using an optical fiber phosphorescence quenching method (Ocean Optics, Inc., Dunedin, FL). The cardiomyocytes were paced to beat by field stimulation $(5 \mathrm{~ms} ; 0.5 \mathrm{~Hz}$, Warner Instruments Inc.).

In all experiments, cardiomyocytes were perfused and equilibrated with the Tyrode's modified solution for $15 \mathrm{~min}$. To simulate ischemia, the perfusion was stopped and cardiomyocytes were exposed for varying duration $(0.5,1,2$ and $3 \mathrm{~h})$ to a hypoxic medium maintaining $\mathrm{pO}_{2}<1 \%$. This medium consisted of a glucosefree Tyrode's modified solution ( $\mathrm{pH}=7.4)$ supplemented with $20 \mathrm{mM}$ of 2- 
deoxyglucose and bubbled with $100 \% \mathrm{~N}_{2}$. In some experiments, Tyrode's modified solution was adjusted to $\mathrm{pH}=6.4$. The gas mixture infused into the chamber was replaced by a constant stream of $\mathrm{N}_{2}(100 \%)$. At the end of the ischemic period, reoxygenation was induced by restoring rapidly the Tyrode's flow and $21 \% \mathrm{O}_{2}$ in the chamber.

In separate experiments, cardiomyocytes were treated either with cyclosporin A $(1 \mu \mathrm{M}$; Sigma) or with antioxidants mixture containing 2-mercaptopropionylglycine

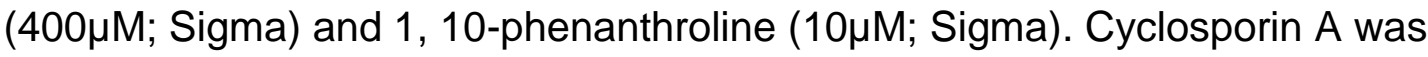
introduced $15 \mathrm{~min}$ before the end of hypoxia and maintained during the first $10 \mathrm{~min}$ of reoxygenation while antioxidants were added according to 3 different experimental protocols: (1) throughout the experiment, (2) during hypoxia or (3) 15 min before the end of hypoxia and during the first $10 \mathrm{~min}$ of reoxygenation.

\section{3. mPTP opening and cell death in cardiomyocytes.}

Direct assessment of mPTP opening in cardiomyocytes was made by means of the established loading procedure of the cells with calcein acetoxymethyl ester (calcein-AM) and $\mathrm{CoCl}_{2}$ (Sigma) resulting in mitochondrial localization of calcein fluorescence (Petronilli et al., 1999). Briefly, cells were loaded with $1 \mu \mathrm{M}$ calcein$\mathrm{AM}$ at $37^{\circ} \mathrm{C}$ for $30 \mathrm{~min}$ in $\mathrm{M} 199$ containing a mixture of insulin-transferrine-selenium $(100 \mu \mathrm{l}$, Sigma). Calcein-AM generates free fluorescent intracellular calcein upon cleavage of the ester bound. $\mathrm{CoCl}_{2}$ was then added to the medium resulting in quenching of cytosolic calcein fluorescence while intramitochondrial calcein allows visualization of mitochondria as bright fluorescent bodies. Subsequently, cardiomyocytes were washed free of calcein-AM and $\mathrm{CoCl}_{2}$ and incubated in the Tyrode's modified solution. 
To determine cell death, cardiomyocytes were co-loaded with propidium iodide (PI, 2.5 $\mu \mathrm{M}$; Sigma) which permeates only the damaged cells (Lemasters et al., 1987).

\subsection{Detection of intracellular oxidant generation.}

Intracellular oxidants were evaluated by measuring changes in fluorescence of two intracellular fluorescent probes, dihydroethidium (DHE, $5 \mu \mathrm{M}$; Molecular probes

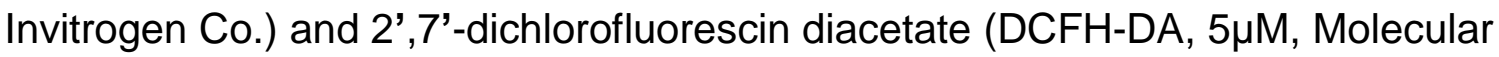
probes, Invitrogen Co.). In distinct experiments, cardiomyocytes were equilibrated for $15 \mathrm{~min}$ with the Tyrode's modified solution containing DHE or DCFH-DA to achieve loading of the fluorochromes, which were also added to the hypoxic and reoxygenation media.

DHE permeates cell membrane easily and can be directly oxidized by reactive oxygen species, more specifically here superoxide anion $\left(\mathrm{O}_{2}{ }^{--}\right)$(Vanden Hoek et al., 1997; Lam et al., 2011) to red fluorescent ethidium which is trapped in the nucleus by intercalation into DNA (Eth-DNA), further amplifying its fluorescence (Laurindo et al., 2008). DCFH-DA enters the cells where esterases cleave the acetate group, trapping the reduced probe (DCFH) intracellularly. The latter is readily oxidized by reactive oxygen species, particularly hydrogen peroxide $\left(\mathrm{H}_{2} \mathrm{O}_{2}\right)$ and hydroxyl radical ("OH) (Vanden Hoek et al., 1997; Possel et al., 1997; Wang et al., 2007) yielding to the fluorescent product dichlorofluorescein (DCF).

\subsection{Data acquisition and analysis.}

The perfusion chamber was mounted on an Olympus IX-81 motorized inverted fluorescent microscope equipped with a mercury lamp as a source of light for epifluorescent illumination, a shutter, filter wheel and appropriate excitation and emission filters. For detection of calcein fluorescence, a 460 to $490 \mathrm{~nm}$ excitation and a $510 \mathrm{~nm}$ emission filters were used. PI fluorescence was excited at 520 to 
$550 \mathrm{~nm}$ and recorded at $580 \mathrm{~nm}$. Eth-DNA fluorescence was measured using 545 $\mathrm{nm}$ excitation and $605 \mathrm{~nm}$ emission filters while DCF fluorescence was detected using $495 \mathrm{~nm}$ and $520 \mathrm{~nm}$ wavelengths for excitation and emission, respectively. Fluorescent images were acquired using a 12-bit cooled CC12 Hamamatsu slowscanning PC-controlled camera (Hamamatsu, Hamamatsu city, Japan). Data were obtained and analyzed using Cell-M software (Olympus, Rungis, France). In some experiments, images were acquired every minute during both hypoxia and reoxygenation periods. For all other experiments, images were obtained every $15 \mathrm{~min}$ during the first $135 \mathrm{~min}$ of the experiment (end of hypoxia). During reoxygenation, images were registered every $5 \mathrm{~min}$ until the end of the protocol for DHE experiments while for DCF, images were recorded every min throughout the first $5 \mathrm{~min}$ and then every $5 \mathrm{~min}$ for the remaining duration of the experiment. Fluorescence was integrated over a region of interest $\left(\sim 80 \mu m^{2}\right)$ for each cardiomyocyte and the background was identified as an area without cells. For each experiment, we calculated the global response observed by averaging the fluorescence changes obtained from all the cardiomyocytes in the field (40-50 cells). The curves presented in the figures correspond to the mean of 3-6 independent experiments.

For calcein experiments, intensity values were normalized according to the initial fluorescence values after subtracting background. Moreover, we calculated the average time to $\mathrm{mPTP}$ opening ( $\mathrm{T}_{50 \mathrm{mPTP}}$ ) in each experiment by measuring the reoxygenation time necessary to reach $50 \%$ decrease in calcein fluorescence intensity for each cell with opened mPTP in the field.

PI fluorescence intensity minus background was normalized to $100 \%$ cell death, as obtained at the end of each experiment after addition of Triton (10\%) to 
permeabilize all cells and the average time for $50 \%$ cell death $\left(\mathrm{T}_{50} \mathrm{PI}\right)$ was calculated.

Intensity values of Eth-DNA and DCF, after background subtraction and normalization according to the initial fluorescence value were either listed as arbitrary units or reported as percent of the maximum value achieved during control experiments.

\subsection{Statistical analysis.}

Results are reported as means \pm S.E.M. Statistical significance was determined using one-way ANOVA for $\mathrm{T}_{50} \mathrm{mPTP}$ and $\mathrm{T}_{50} \mathrm{PI}$ and a two-way ANOVA for intensity profiles followed in all cases by student's t-test. Significance was accepted when $P<0.05$

\section{Results:}

\section{1. mPTP opening and cell death in adult rat cardiomyocytes subjected to} hypoxia/reoxygenation depends on the duration of hypoxia.

Fig.1A illustrated the decrease in calcein fluorescence, representing mPTP opening in adult cardiomyocytes loaded with calcein and $\mathrm{CoCl}_{2}$ and subjected to hypoxia/reoxygenation as a function of the duration of exposure to hypoxia. Compared with normoxic cells perfused with Tyrode's modified solution under $21 \% \mathrm{O}_{2}$ for $5 \mathrm{~h}$, cardiomyocytes subject to 1,2 and $3 \mathrm{~h}$ of hypoxia, but not $0.5 \mathrm{~h}$ showed a significant decrease in calcein fluorescence during reoxygenation. This decrease was more rapid when the duration of hypoxia was increased.

Consequently, $\mathrm{T}_{50} \mathrm{mPTP}$ (Fig.1B) and the percentage of cells with non-opened mPTP (cardiomyocytes that maintained their calcein fluorescence at the end of reoxygenation, Fig.1C) decreased with longer duration of hypoxia. In fact, after $1 \mathrm{~h}$ 
of hypoxia $10.20 \pm 0.92 \%$ of cardiomyocytes maintained their calcein fluorescence and hence their mPTP closed at the end of the reoxygenation period, but all of them totally lost this fluorescence following both 2 and 3h of hypoxia (Fig.1C). However, we observed that $2 \mathrm{~h}$ of hypoxia without reoxygenation had no effect on the decrease in calcein fluorescence whereas $14.29 \pm 0.22 \%$ of cardiomyocytes had already lost their calcein fluorescence after 3h of hypoxia. For these reasons, $2 \mathrm{~h}$ was considered as the optimal duration of hypoxia to be used all over this study. Since no significant evolution in calcein fluorescence intensity was observed during $2 \mathrm{~h}$ of hypoxia, all the figures illustrating the changes in calcein fluorescence intensity displayed only the variations during reoxygenation, (i.e., equilibration and hypoxic periods were omitted for clarity). Once the time of hypoxia has been fixed, sequential cardiomyocytes mortality was quantified by cell staining with PI. When the cardiomyocytes were co-loaded with calcein, $\mathrm{CoCl}_{2}$ and $\mathrm{PI}$, the decrease in calcein fluorescence during reoxygenation was coupled with a simultaneous increase in nuclear labeling by $\mathrm{PI}$, indicating plasma membrane permeabilization and cell death. As illustrated in Fig.2A, after $2 \mathrm{~h}$ of hypoxia, $100 \%$ of cell death was attained following $\sim 1 \mathrm{~h}$ of reoxygenation. This loss of viability was preceded by mPTP opening as evaluated by loss in calcein fluorescence. In these conditions, $\mathrm{T}_{50} \mathrm{mPTP}$ was $30.62 \pm 0.06 \mathrm{~min}$ and $\mathrm{T}_{50} \mathrm{PI}$ was $42.50 \pm 0.79 \mathrm{~min}$. Cyclosporin $\mathrm{A}(1 \mu \mathrm{M})$, which is known to inhibit mPTP opening through a direct effect on cyclophilin D and to protect myocardium against ischemia/reperfusion, increased $\mathrm{T}_{50} \mathrm{mPTP}$ by 2 fold, from $30.62 \pm 0.66 \mathrm{~min}$ to $64.8 \pm 1.36 \mathrm{~min}(p<0.05$, Fig.2B) and the percentage of cells with non-opened mPTP at the end of reoxygenation from $1.58 \pm 0.03 \%$ to $22.09 \pm 0.53 \%(P<0.05)$. In addition, cyclosporin A concomitantly delayed and prevented nuclear staining by $\mathrm{PI}\left(\mathrm{T}_{50} \mathrm{Pl}\right.$ : 
$110.5 \pm 2.69 \mathrm{~min})$, indicating that cyclosporin A also reduced hypoxia/reoxygenationinduced cell death.

Interestingly, on each experiment, we observed two types of calcein fluorescence signal kinetics among cardiomyocytes in the same field (Fig.3A). Some cells exhibited an abrupt ( 2min) and complete drop of calcein fluorescence, while others showed a gradual and slow decrease in this fluorescence. These observations are consistent with two modes of mPTP opening i.e, transient (reversible) and long-lasting (irreversible) as previously described (Petronilli et al., 1999; Sharov et al., 2007). However, the dependence of these modes of mPTP opening on the duration of hypoxia is described to our knowledge for the first time in the present study. As shown in Fig.3B, the percentage of cardiomyocytes displaying a rapid mPTP opening increased on the detrimental of those presenting a slow opening when lengthening the duration of the hypoxic insult. Treatment of cardiomyocytes with $1 \mu \mathrm{M}$ cyclosporin $\mathrm{A}$ also changed the dynamic of calcein efflux. Indeed, cyclosporin A increased by $58 \pm 3 \%$ the cells displaying a gradual process as compared to control while the percentage of cells exhibiting rapid mPTP opening decreased by $28 \pm 3 \%$ as compared to control confirming the involvement of mPTP opening in this phenomenon.

\subsection{Reactive oxygen species production during hypoxia plays a key role on mPTP opening at reoxygenation.}

In separate experiments, cardiomyocytes were co-loaded either with DHE, calcein$\mathrm{AM}$ and $\mathrm{CoCl} 2$ or with $\mathrm{DCFH}-\mathrm{DA}$ and $\mathrm{PI}$ and reactive oxygen species production was assessed after 0.5, 1 and $2 \mathrm{~h}$ of hypoxia. As shown in Table 1, significant increases in both Eth-DNA and DCF fluorescence were detected when increasing 
the duration of hypoxia. These experiments revealed a 4 fold increase in Eth-DNA and 6 fold increase in DCF production at the end of $2 \mathrm{~h}$ hypoxia as compared to $0.5 \mathrm{~h}$ of hypoxia, respectively $(\mathrm{P}<0.05)$. A rapid increase in bothfluorescence was observed during the last $15 \mathrm{~min}$ of hypoxia when cardiomyocytes were subjected to $2 \mathrm{~h}$ of hypoxia (Fig 4). We have no clear explanation for this effect but Guarnieri et al. (1980) have demonstrated that hypoxia induced loss of cellular components of the antioxidant system protecting cells from reactive oxygen species formation. This may create conditions accelerating reactive oxygen species production at the end of the hypoxic period.

A large decrease in Eth-DNA fluorescence intensity was associated with the first minutes of reoxygenation. DCF fluorescence increased rapidly and transiently within the first minutes of reoxygenation ( 20\% increase within $5 \mathrm{~min}$ of reoxygenation as compared to the corresponding levels at the end of hypoxia, $\mathrm{P}<0.05)$. Then, the fluorescence decreased progressively which could represent either escape of the oxidized probe from the cell, possibly as a result of cell death, or intracellular reduction of the oxidized probe (Duranteau et al., 1998).

To determine the significance and link between the oxidant stress during hypoxia and MPTP opening at reoxygenation, cardiomyocytes were subjected to hypoxia/reoxygenation in the presence of antioxidant agents (association of $400 \mu \mathrm{M} 2$-mercaptopropionylglycine and $10 \mu \mathrm{M}$ phenanthroline, effective concentrations of these antioxidants reported previously (Becker et al., 1999)) administered either throughout the experiment, or during reoxygenation, or during hypoxia. These antioxidants were chosen for their specific activity against ${ }^{\circ} \mathrm{OH}$ and $\mathrm{H}_{2} \mathrm{O}_{2}$. Indeed, 2-mercaptopropionylglycine, a cell permeable synthetic glutathione analog enhances the scavenging of $\mathrm{H}_{2} \mathrm{O}_{2}$ (Bolli et al., 1998), and has a slight effect 
as a superoxide scavenger (Dikalov et al., 1996). phenanthroline binds ferrous iron and thus reduces its availability as a catalyst in the Fenton reaction, responsible for ${ }^{\circ} \mathrm{OH}$ formation (Thomas et al., 2009).

As shown in Fig.4A, cardiomyocytes exhibited significantly less Eth-DNA fluorescence at the end of the ischemic challenge when they were treated with antioxidants administered either throughout the experiment $(60 \pm 7 \%$ vs. $100 \pm 2 \%$ in control, $\mathrm{P}<0.05)$ or during hypoxia $(58 \pm 6 \%$ vs. $100 \pm 2 \%$ in control, $\mathrm{P}<0.05)$. Control experiments were associated with an initial abrupt drop in Eth-DNA fluorescence during the first minutes of reoxygenation followed by a constant decrease in this fluorescence, also observed when antioxidants were added at reoxygenation. In contrast, antioxidants introduced throughout the experiment or during hypoxia prevented the initial abrupt drop in Eth-DNA fluorescence during the first minutes of reoxygenation and maintained at a higher level this fluorescence as compared to control. As shown in Fig.4B, increases in DCF fluorescence during hypoxia and reoxygenation were significantly attenuated by addition of antioxidants either throughout the experiment or during hypoxia but not when added only at reoxygenation. Knowing that DCFH could be oxidized by intracellular heme compounds, we evaluated the effect of phenanthroline alone on the DCF signal during hypoxia. We observed that phenanthroline slightly decreased DCF signal at the end of hypoxia as compared to control (data not shown). Therefore, DCF signal observed during hypoxia is related to the reaction of $\mathrm{DCFH}$ with reactive oxygen species and not to the oxidation of DCFH by heme.

These variations in reactive oxygen species generation in the presence of antioxidants were associated with concomitant changes in calcein and PI 
fluorescence. As compared with control experiments, cells treated with antioxidants throughout the experiment or during hypoxia demonstrated a significant right shift of the curves representing the decrease in calcein fluorescence at reoxygenation (Fig.5A). Accordingly, $\mathrm{T}_{50} \mathrm{mPTP}$ was significantly delayed, while antioxidants introduced at reoxygenation had no effect on the time for mPTP opening. In a parallel manner, antioxidants administered throughout the experiment or during hypoxia partially prevented cell death as shown by PI labeling (fig.5B). Consequently, $\mathrm{T}_{50} \mathrm{PI}$ as well as the percentage of cells with nonopened $\mathrm{mPTP}$ at the end of $2 \mathrm{~h}$ reoxygenation were significantly increased in these conditions (table 2).

These findings led us to conclude that ischemic oxidant stress level reached after $2 \mathrm{~h}$ of hypoxia is necessary to prime the cardiomyocytes for mPTP opening and to induce their death at reoxygenation. By preventing reactive oxygen species generation during hypoxia, antioxidants therefore protect the cells against the reactive oxygen species burst at reoxygenation and prevent both mPTP opening and cell death.

\subsection{Cyclosporin A, mPTP opening and reactive oxygen species production during hypoxia/reoxygenation.}

As mentioned before (Fig.2), when cardiomyocytes were reoxygenated in the presence of cyclosporin $A$, calcein was retained by mitochondria, $T_{50} \mathrm{mPTP}$ increased and cell death was delayed as compared to control. The effect of cyclosporin A on reactive oxygen species formation was also evaluated. During hypoxia and in the presence of cyclosporin A, oxidation of both DHE and DCFH were comparable to control levels (Fig.6 and 7). As illustrated in Fig.6, cyclosporin 
A at reoxygenation did not prevent the abrupt drop in Eth-DNA fluorescence during the first minutes of reoxygenation but maintained Eth-DNA fluorescence thereafter. Moreover, DCF fluorescence displayed a biphasic pattern of reactive oxygen species production at reoxygenation in the presence of cyclosporin A (Fig.7) i.e, a similar increase in DCF fluorescence was observed in the presence or in the absence of cyclosporin A during the first 10 min of reoxygenation but this fluorescence was shifted to the left thereafter, in the presence of cyclosporin $A$ as compared to control. Taken together, these results suggest that cyclosporin A maintained $\mathrm{O}_{2}{ }^{--}$production and decreased ${ }^{\circ} \mathrm{OH}$ and/or $\mathrm{H}_{2} \mathrm{O}_{2}$ formation during reoxygenation.

\section{Discussion:}

The present study established a cause-effect relationship between the reactive oxygen species produced during hypoxia/reoxygenation, mPTP opening and subsequent cell death at reoxygenation and emphasized the major importance of $\cdot \mathrm{OH}$ and/or $\mathrm{H}_{2} \mathrm{O}_{2}$ production throughout hypoxia on this effect.

To achieve these conclusions, we used a model of isolated beating adult rat cardiomyocytes subjected to hypoxia/reoxygenation to evaluate repeatedly and simultaneously as a function of time mPTP opening, cell death and/or reactive oxygen species production. These cardiomyocytes were exposed to an environment as close as possible to that experienced by cardiac cells during myocardial ischemia/reperfusion. It is closer to this pathological situation than other models using TMRM photoexcitability (Hausenloy et al., 2004; Ruiz-Meana et al., 2007) permeabilization of cellular membrane in the presence of high $\mathrm{Ca}^{2+}$ concentration (Seguchi et al., 2005) or exposure of cells to pro-oxidant agents 
(Park et al., 2006). Furthermore, this model depends on contractile activity of the cells as neither MPTP opening nor cell death was observed in the absence of electrical stimulation. A plausible explanation is that the part of metabolic cost for contractile activity involves $\mathrm{Ca}^{2+}$ and glucose availability (Haworth et al., 1983; Geisbuhler et al., 1983). It has been demonstrated that reducing oxygen and glucose concentrations of the hypoxic perfusate resulted in poorer recovery of the cardiomyocytes (Geisbuhler et al., 1990; Poizat et al., 1994). This led us to use a metabolic blocker, 2-deoxy-D-glucose to limit glucose supply available to the cells. As cardiomyocytes maintained under normoxic conditions for $\sim 5$ h showed no significant changes in calcein fluorescence, the observed response to hypoxia/reoxygenation cannot be explained by a time-effect. Furthermore, this study showed that the duration of hypoxia was proportional to the percentage of cells exhibiting mPTP opening and cell death at the end of reoxygenation. However, hypoxia alone was not sufficient to induce mPTP opening. Our data showed that MPTP opening precedes cell death confirming that it is a major causative event for cell death. In addition, we observed that mPTP opening occurs at least with two different kinetic modes, i.e., rapid and slow openings already reported by other investigators (Griffiths et al., 2000; Kim et al., 2006). The rapid kinetic phase is usually accompanied by a phase of cell shortening associated with a rapid and full loss of calcein fluorescence and it corresponds to a long lasting opening of MPTP then followed by a rapid cell death. The slow kinetic phase corresponds to a transient opening of MPTP and consists in a slow and gradual loss of calcein fluorescence at reoxygenation not followed by a rapid cell death that occurred only when mPTP opening became sustained. Kim et al. 
(2006) associated this transient opening to a partial depletion of ATP leading to inhibition of $\mathrm{Ca}^{2+}$ cellular pumps.

A major result of the present study is the demonstration that reactive oxygen species produced during hypoxia play a crucial and necessary role for mPTP opening and hypoxia/reoxygenation related injury. This is not in conflict with several studies showing that a burst of oxidant occurs during the first few minutes after reperfusion of the ischemic myocardium (Ambrosio et al., 1993; Flaherty and Weisfeldt, 1998; Piper et al., 2004) but it shows that the oxidant stress begins during hypoxia prior to the onset of reoxygenation. However, the immediate effects of this oxidant stress are not lethal since cardiac cell death does not increase during hypoxia. It seems that reactive oxygen species produced during hypoxia predispose cardiomyocytes to reoxygenation injury through their progressive depletion of antioxidant capacity (Ceconi et al., 2000).

The use of two different intracellular probes (DHE and DCFH-DA) permitted us to evaluate which reactive oxygen species were more specifically involved in cardiomyocytes injury during hypoxia/reoxygenation. Oxidation of DCFH is indicative of $\mathrm{H}_{2} \mathrm{O}_{2}$ or ${ }^{\circ} \mathrm{OH}$ formation, while oxidation of DHE provided a measure of $\mathrm{O}_{2}{ }^{--}$formation (Vanden Hoek et al., 1997; Dikalov et al., 2007). It should be noted that a severe production of ${ }^{\circ} \mathrm{OH}$ can affect Eth-DNA complex stability and decreases fluorescence (Vanden Hoek et al., 1997). In line with this, we observed an abrupt drop in the Eth-DNA fluorescence coupled with $\mathrm{H}_{2} \mathrm{O}_{2}$ and ${ }^{\circ} \mathrm{OH}$ production during the first few minutes of reoxygenation as attested by a transient increase in DCF fluorescence. Moreover, our experiments showed that significant levels of reactive oxygen species, more particularly $\mathrm{H}_{2} \mathrm{O}_{2}$ and ${ }^{\circ} \mathrm{OH}$ were generated during hypoxia prior to reoxygenation. This might be paradoxical, as the ischemic 
conditions include a low $\mathrm{pO}_{2}$ that might limit the production of oxyradicals, but increasing number of evidence supports this concept (Becker et al., 1999; Robin et al., 2007; Duranteau et al., 1998). One explanation could be that highly reduced components of the electron transfer chain promote electron leak to residual $\mathrm{O}_{2}$ resulting in reactive oxygen species production (Opie, 1989) which can be accentuated by the loss of cellular components of the antioxidant system. In this regard, the present study confirms that increasing the time of hypoxia is directly related to an increasing oxidant generation, even before reoxygenation, leading to a more intense reoxygenation injury represented here by MPTP opening and cell death.

Two lines of evidence support here the important role of reactive oxygen species generation during hypoxia in cardiomyocytes injury. First, when antioxidants preferentially targeting against $\mathrm{H}_{2} \mathrm{O}_{2}$ and ${ }^{\circ} \mathrm{OH}$ are available during the hypoxic period, the production of these species is inhibited during this period, as detected by DCF. Superoxide anion generation was also slightly lowered, more particularly during the last $15 \mathrm{~min}$ of hypoxia. Furthermore, antioxidants available during hypoxia blunted the abrupt fall in Eth-DNA fluorescence detected during the first minutes of reoxygenation probably due to less ${ }^{\circ} \mathrm{OH}$ generation as illustrated by DCF signal reduction. This was accompanied by a significant delay for mPTP opening and decrease in cell death, as reflected by the rise in the percentage of cells with non-opened mPTP. In contrast to the decreased production of ${ }^{\circ} \mathrm{OH}$ and $\mathrm{H}_{2} \mathrm{O}_{2}$, the delay in mPTP opening was associated with maintenance of $\mathrm{O}^{-{ }_{2}}$ production at reoxygenation similar to the level observed in cells under normoxic conditions (data not shown). This is in accordance with observations of other investigators who described $\mathrm{O}^{--}{ }_{2}$ production in mitochondria and cardiomyocytes 
under normal conditions and during reoxygenation following hypoxia (Sheu et al., 2008; Wang et al., 2008). Thus, this $\mathrm{O}_{2}{ }^{--}$production, that accompanied mPTP inhibition, may reflect a better functioning of the respiratory chain and thus cell viability, suggesting a cardioprotective effect. Second, antioxidants administered $15 \mathrm{~min}$ before the end of hypoxia and during the first $10 \mathrm{~min}$ of reoxygenation neither interfered with the profile of oxidant generation nor affected the time for mPTP opening. Therefore, reactive oxygen species production during hypoxia is necessary for promoting the reoxygenation burst. This absence of effect of antioxidants added few minutes before reoxygenation could be due to the lack of sufficient time to act before the reoxygenation burst. In either case, these findings reinforce the importance of the oxidant stress during hypoxia. It is conceivable that hypoxic reactive oxygen species predispose the cells for reoxygenation stress by eroding cellular antioxidants defenses, thereby leaving the cell more vulnerable to oxidant generation at reoxygenation (Becker, 2004; Robin et al., 2007). The present study demonstrates that hypoxic reactive oxygen species directly triggers or prime the cells for mPTP opening at reoxygenation. It should be noted that increased reactive oxygen species production is not the only mechanism to explain damage upon reperfusion. In fact, cellular damages caused by ischemia/reperfusion probably result from multiple factors (Yellon and Hausenloy, 2007). However, this study demonstrates that reactive oxygen species produced during hypoxia, and not only those produced at reperfusion, are a major contributor to the damages observed at reperfusion, represented here by MPTP opening. This conclusion is consistent with previous observations that protective strategies like pre-conditioning (Di Lisa et al., 2007; Peterson et al., 2011) and therapeutic hypothermia (Riess et al., 2004; Tissier et al., 2010) confer their 
protection by attenuating reactive oxygen species production during ischemia. It would also explain why clinical trials using antioxidants (Farbstein et al., 2010) failed to show clear benefit when treatments are administered only at reperfusion. Finally, cyclosporin A and other molecules interacting with cyclophilin-D can inhibit mPTP opening directly, independently of reactive oxygen species generation (Halestrap et al., 2004; Kim et al., 2006). In the present study, cyclosporin A prevented mPTP opening despite reactive oxygen species formation during hypoxia and increased the percentage of cardiomyocytes that displayed gradual mPTP opening on the detriment of those with rapid opening. We observed the same profiles of mPTP opening in the presence of cardioprotective agents acting through other pharmacological mechanisms such as GSK-3ß inhibition (Obame et al., 2008) or Translocator protein modulation (Schaller et al., 2010). In this regard, $\mathrm{O}_{2}-{ }_{2}$ production during reoxygenation under cyclosporin $\mathrm{A}$ was comparable to that observed with antioxidants reflecting also an improvement in cell viability. However, neither the decrease in Eth-DNA nor the increases in DCF during the first minutes of reoxygenation were attenuated by cyclosporin A. Taken together, these results support the conclusion that cyclosporin A delays mPTP opening and cell death without interfering with oxidant generation during hypoxia/reoxygenation.

As with all in vitro models, the current results should be interpreted within the constraints of limitations and may not be directly comparable with those obtained in vivo. One of these limitations is the lack of factors that in vivo pathological conditions affect cardiomyocyte function such as inflammatory mediators or reactive oxygen species produced by other types of cells. These factors are 
absent in the isolated cardiomyocyte model and may contribute to increase the damage at reperfusion in intact hearts.

\section{Conclusions}

This study emphasizes the role and the link between reactive oxygen species production during hypoxia and mPTP opening and subsequent cell death at reoxygenation. We demonstrated that increasing the time of hypoxia is directly related to increasing oxidant generation and that production of ${ }^{\circ} \mathrm{OH}$ and $\mathrm{H}_{2} \mathrm{O}_{2}$ during hypoxia is necessary to induce mPTP opening and cell death at reoxygenation. Additional understanding of the role of ischemic oxidant generation is necessary for the development of appropriate biomarkers to predict the outcome in acute myocardial infarction in patients (Lorgis et al., 2010) and treatment strategies to limit ischemia/reperfusion injuries. 


\section{Acknowledgments:}

R. Assaly and S. Paradis were supported by doctoral grants from the Region lle de France and the Ministère de la Recherche et de la Technologie, respectively. The authors wish to thank Clement Mompied for his technical support. This work was supported in part by a grant of the Fondation de France (²009002496). 


\section{References:}

Adlam, V.J., Harrison, J.C., Porteous, C.M., James, A.M., Smith, R.A., Murphy, M.P., Sammut, I.A., 2005. Targeting an antioxidant to mitochondria decreases cardiac ischemiareperfusion injury. Faseb J. 19, 1088-1095.

Ambrosio, G., Zweier, J.L., Duilio, C., Kuppusamy, P., Santoro, G., Elia, P.P., Tritto I.; Cirillo, P., Condorelli, M., Chiariello, M., Flaherty, J.T., 1993. Evidence that mitochondrial respiration is a source of potentially toxic oxygen free radicals in intact rabbit hearts subjected to ischemia and reflow. J. Biol. Chem. 268, 18532-18541.

Becker, L.B., Vanden Hoek, T.L., Shao, Z.H., Li, C.Q., Schumacker, P.T., 1999. Generation of superoxide in cardiomyocytes during ischemia before reperfusion. Am. J. Physiol. 277, H2240-H2246.

Becker, L.B., 2004. New concepts in reactive oxygen species and cardiovascular reperfusion physiology. Cardiovasc. Res. 61, 461-470.

Bolli, R., Patel, B.S., Jeroudi, M.O., Lai, E.K., McCay, P.B., 1998. Demonstration of free radical generation in "stunned" myocardium of intact dogs with the use of the spin trap alpha-phenyl N-tert-butyl nitrone. J. Clin. Invest. 82, 476-485.

Ceconi, C., Bernocchi, P., Boraso, A., Cargnoni, A., Pepi, P., Curello, S., Ferrari, R., 2000. New insights on myocardial pyridine nucleotides and thiol redox state in ischemia and reperfusion damage. Cardiovasc. Res. 47, 586-594. 
Dikalov, S., Khramtsov, V., Zimmer, G., 1996. Determination of rate constants of the reactions of thiols with superoxide radical by electron paramagnetic resonance: critical remarks on spectrophotometric approaches. Arch. Biochem. Biophys. 326, 207-218.

Dikalov, S., Griendling, K.K., Harrison, D.G., 2007. Measurement of reactive oxygen species in cardiovascular studies. Hypertension 49, 717-727.

Di Lisa, F., Canton, M., Menabo, R., Kaludercic, N., Bernardi, P., 2007. Mitochondria and cardioprotection. Heart Fail. Rev. 12, 249-260.

Di Lisa, F., Bernardi, P. A., 2009. CaPful of mechanisms regulating the mitochondrial permeability transition. J. Mol. Cell. Cardiol. 46, 775-780.

Duranteau, J., Chandel, N.S., Kulisz, A., Shao, Z., Schumacker, P.T. 1998. Intracellular signaling by reactive oxygen species during hypoxia in cardiomyocytes. J. Biol. Chem. $273,11619-11624$.

Farbstein, D., Kozak-Blickstein, A., Levy, A.P., 2010. Antioxidant vitamins and their use in preventing cardiovascular disease. Molecules 15, 8098-8110.

Flaherty, J.T., Weisfeldt, M.L., 1998. Reperfusion injury. Free Radic. Biol. Med. 5, 409419.

Guarnieri, C., Flamigni, F., Caldarera, C.M., 1980. Role of oxygen in the cellular damage induced by re-oxygenation of hypoxic heart. J. Mol. Cell. Cardiol. 12, 797-808. 
Geisbuhler, T., Altschuld, R.A., Trewyn, R.W., Ansel, A.Z., Lamka, K., Brierley, G.P., 1984. Adenine nucleotide metabolism and compartmentalization in isolated adult rat heart cells. Circ. Res. 54, 536-546.

Geisbuhler, T.P., Rovetto, M.J., 1990. Lactate does not enhance anoxia/reoxygenation damage in adult rat cardiac myocytes. J. Mol. Cell. Cardiol. 22, 1325-1235.

Griffiths, E.J., Ocampo, C.J., Savage, J.S., Stern, M.D., Silverman, H.S., 2000. Protective effects of low and high doses of cyclosporin A against reoxygenation injury in isolated rat cardiomyocytes are associated with differential effects on mitochondrial calcium levels. Cell. Calcium 27, 87-95.

Halestrap A.P., Clarke, S.J., Javadov, S.A., 2004. Mitochondrial permeability transition pore opening during myocardial reperfusion--a target for cardioprotection. Cardiovasc. Res. 61, 372-385.

Halestrap, A.P., 2009. What is the mitochondrial permeability transition pore? J. Mol. Cell. Cardiol. 46, 821-831.

Hausenloy, D.J., Yellon, D.M., Mani-Babu, S., Duchen, M.R., 2004. Preconditioning protects by inhibiting the mitochondrial permeability transition. Am. J. Physiol. Heart Circ. Physiol. 287, H841-H849.

Haworth, R.A., Hunter, D.R., Berkoff, H.A., Moss, R.L., 1983. Metabolic cost of the stimulated beating of isolated adult rat heart cells in suspension. Circ. Res. 52, 342-351. 
Javadov, S.A., Clarke, S., Das, M., Griffiths, E.J., Lim, K.H., Halestrap, A.P., 2003. Ischaemic preconditioning inhibits opening of mitochondrial permeability transition pores in the reperfused rat heart. J. Physiol. 549, 513-524.

Kim, J.S., Jin, Y., Lemasters, J.J., 2006. Reactive oxygen species, but not Ca2+ overloading, trigger $\mathrm{pH}$ - and mitochondrial permeability transition-dependent death of adult rat myocytes after ischemia-reperfusion. Am. J. Physiol. Heart Circ. Physiol. 290, H2024-H2034.

Kowaltowski, A.J., Castilho, R.F., Vercesi, A.E., 2001. Mitochondrial permeability transition and oxidative stress. FEBS Lett. 495, 12-15.

Lam, Y.T., Aung-Htut, M.T., Lim, Y.L., Yang, H., Dawes, I.W., 2011. Changes in reactive oxygen species begin early during replicative aging of Saccharomyces cerevisiae cells. Free Radic. Biol. Med. 50, 963-970.

Laurindo, F., Fernandes, D.C., Santos, C.X., 2008. Assessment of superoxide production and NADPH oxidase activity by HPLC analysis of dihydroethidium oxidation products. Methods Enzymol. 441, 237-260.

Lemasters, J.J., DiGuiseppi, J., Nieminen, A.L., Herman, B., 1987. Blebbing, free Ca2+ and mitochondrial membrane potential preceding cell death in hepatocytes. Nature 325 , $78-81$.

Lorgis, L., Zeller, M., Dentan, G., Sicard, P., Richard, C., Buffet, P., L'Huillier, I., Beer, J.C., Cottin, Y., Rochette, L., Vergely, C., 2010. The free oxygen radicals test (FORT) to 
assess circulating oxidative stress in patients with acute myocardial infarction.

Atherosclerosis 213, 616-621.

Morin, D., Assaly, R., Paradis, S., Berdeaux, A., 2009. Inhibition of mitochondrial membrane permeability as a putative pharmacological target for cardioprotection. Curr. Med. Chem. 16, 4382-4398.

Murphy, E., Steenbergen, C., 2008. Mechanisms underlying acute protection from cardiac ischemia-reperfusion injury. Physiol. Rev. 88, 581-609.

Obame, F.N., Plin-Mercier, C., Assaly, R., Zini, R., Dubois-Rande, J.L., Berdeaux, A., Morin, D., 2008. Cardioprotective effect of morphine and a blocker of glycogen synthase kinase 3 beta, SB216763 [3-(2,4-dichlorophenyl)-4(1-methyl-1H-indol-3-yl)-1H-pyrrole-2,5dione], via inhibition of the mitochondrial permeability transition pore. J. Pharmacol. Exp. Ther. 326, 252-258.

Opie L.H., 1989. Reperfusion injury and its pharmacologic modification. Circulation 80, $1049-62$.

Ovize, M., Baxter, G.F., Di Lisa, F., Ferdinandy, P., Garcia-Dorado, D., Hausenloy, D.J., Heusch, G., Vinten-Johansen, J., Yellon, D.M., Schulz, R., 2010. Postconditioning and protection from reperfusion injury: where do we stand? Position paper from the Working Group of Cellular Biology of the Heart of the European Society of Cardiology. Cardiovasc. Res. 87, 406-423. 
Park, S.S., Zhao, H., Mueller, R.A., Xu, Z., 2006. Bradykinin prevents reperfusion injury by targeting mitochondrial permeability transition pore through glycogen synthase kinase 3beta. J. Mol. Cell. Cardiol. 40, 708-716.

Peterson, K.M., Aly, A., Lerman, A., Lerman, L.O., Rodriguez-Porcel, M., 2011. Improved survival of mesenchymal stromal cell after hypoxia preconditioning: Role of oxidative stress. Life Sci. 88, 65-73.

Petronilli, V., Miotto, G., Canton, M., Brini, M., Colonna, R., Bernardi, P., Di Lisa, F., 1999. Transient and long-lasting openings of the mitochondrial permeability transition pore can be monitored directly in intact cells by changes in mitochondrial calcein fluorescence. Biophys. J. 76, 725-734.

Piper, H.M., Abdallah, Y., Schafer, C., 2004. The first minutes of reperfusion: a window of opportunity for cardioprotection. Cardiovasc. Res. 61, 365-371.

Poizat, C., Keriel, C., Cuchet, P., 1994. Is oxygen supply sufficient to induce normoxic conditions in isolated rat heart? Basic Res. Cardiol. 89, 535-844.

Possel, H., Noack, H., Augustin, W., Keilhoff, G., Wolf, G., 1997. 2,7-

Dihydrodichlorofluorescein diacetate as a fluorescent marker for peroxynitrite formation. FEBS Lett. 416, 175-178.

Riess, M.L., Camara, A.K., Kevin, L.G., An, J., Stowe, D.F., 2004. Reduced reactive O2 species formation and preserved mitochondrial $\mathrm{NADH}$ and $[\mathrm{Ca} 2+]$ levels during short-term 17 degrees $C$ ischemia in intact hearts. Cardiovasc. Res. 61, 580-590. 
Robin, E., Guzy, R.D., Loor, G., Iwase, H., Waypa, G.B., Marks, J.D., Hoek, T.L., Schumacker, P.T., 2007. Oxidant stress during simulated ischemia primes cardiomyocytes for cell death during reperfusion. J. Biol. Chem. 282, 19133-19143.

Ruiz-Meana, M., Abellan, A., Miro-Casas, E., Garcia-Dorado, D., 2007. Opening of mitochondrial permeability transition pore induces hypercontracture in $\mathrm{Ca} 2+$ overloaded cardiac myocytes. Basic Res. Cardiol. 102: 542-552.

Schaller, S., Paradis, S., Ngoh, G.A., Assaly, R., Buisson, B., Drouot, C., Ostuni, M.A., Lacapere, J.J., Bassissi, F., Bordet, T., Berdeaux, A., Jones, S.P., Morin, D., Pruss, R.M., 2010. TRO40303, a new cardioprotective compound, inhibits mitochondrial permeability transition. J. Pharmacol. Exp. Ther. 333 696-706.

Seguchi, H., Ritter, M., Shizukuishi, M., Ishida, H., Chokoh, G., Nakazawa, H., Spitzer K.W., Barry, W.H., 2005. Propagation of Ca2+ release in cardiac myocytes: role of mitochondria. Cell. Calcium 38, 1-9.

Sharov, V.G., Todor, A., Khanal, S., Imai, M., Sabbah, H.N., 2007. Cyclosporine A attenuates mitochondrial permeability transition and improves mitochondrial respiratory function in cardiomyocytes isolated from dogs with heart failure. J. Mol. Cell. Cardiol. 42, 150-158.

Sheu, S.S., Wang, W., Cheng, H., Dirksen, R.T., 2008. Superoxide flashes: illuminating new insights into cardiac ischemia/reperfusion injury. Future Cardiol. 4, 551-554. 
Thomas, C., Mackey, M.M., Diaz, A.A., Cox, D.P., 2009. Hydroxyl radical is produced via the Fenton reaction in submitochondrial particles under oxidative stress: implications for diseases associated with iron accumulation. Redox Rep. 14, 102-108.

Tissier, R., Chenoune, M., Ghaleh, B., Cohen, M.V., Downey, J.M., Berdeaux, A., 2010. The small chill: mild hypothermia for cardioprotection? Cardiovasc. Res. 88: 406-414.

Townsend, P.A., Davidson, S.M., Clarke, S.J., Khaliulin, I., Carroll, C.J., Scarabelli, T.M., Knight, R.A., Stephanou, A., Latchman, D.S., Halestrap, A.P., 2007. Urocortin prevents mitochondrial permeability transition in response to reperfusion injury indirectly by reducing oxidative stress. Am. J. Physiol. Heart Circ. Physiol. 293, H928-238.

Vanden Hoek, T.L., Li, C., Shao, Z., Schumacker, P.T., Becker, L.B., 1997. Significant levels of oxidants are generated by isolated cardiomyocytes during ischemia prior to reperfusion. J. Mol. Cell. Cardiol. 29, 2571-2583.

Yellon, D.M., Hausenloy, D.J., 2007. Myocardial reperfusion injury. N. Engl. J. Med. 357, $1121-1135$

Wang, W., Fang, H., Groom, L., Cheng, A., Zhang, W., Liu, J., 2008. Superoxide flashes in single mitochondria. Cell 134, 279-290.

Wang, Q.S., Zheng, Y.M., Dong, L., Ho, Y.S., Guo, Z., Wang, Y.X. 2007., Role of mitochondrial reactive oxygen species in hypoxia-dependent increase in intracellular calcium in pulmonary artery myocytes. Free Radic. Biol. Med. 42, 642-653. 


\section{Figure Legends}

Fig. 1: Duration of hypoxia and mPTP opening.

Adult rat cardiomyocytes were co-loaded with calcein- $\mathrm{AM}$ and $\mathrm{CoCl}_{2}$ and images were collected at 1 -min intervals. After a 15-min equilibration period, cardiomyocytes were exposed to either normoxic conditions or varying duration of hypoxia $(0.5,1,2$ or $3 \mathrm{~h})$ followed by reoxygenation. A: Time courses of the changes in calcein signals during the reoxygenation period as function of different duration of hypoxia. Each experiment was calculated by averaging the fluorescence changes obtained from all cardiomyocytes under the same condition normalized to $100 \%$ (40-45 cardiomyocytes per experiment). The global response per condition was obtained by averaging 3 to 6 experiments (values are means \pm S.E.M). B: Time of reoxygenation necessary to induce a $50 \%$ decrease in calcein fluorescence (time to $50 \% \mathrm{mPTP}$ opening, $\mathrm{T}_{50} \mathrm{mPTP}$ ). For each experiment, the response was analyzed by averaging the fluorescence change obtained from cardiomyocytes that lost their calcein fluorescence at the end of the experiment (cells with opened mPTP) contained in a single field. Each value is the mean $\pm S$.E.M of 3 to 6 experiments performed with different isolated preparations of cardiomyocytes. C: Percentage of cells with non-opened mPTP at the end of varying duration of hypoxia followed by $2 \mathrm{~h}$ of reoxygenation. *, $\mathrm{P}<0.05$ versus hypoxia $0.5 \mathrm{~h} ; \dagger, \mathrm{P}<0.05$ versus hypoxia $1 \mathrm{~h} ; \ddagger, \mathrm{P}<0.05$ versus hypoxia $2 \mathrm{~h}$ by ANOVA followed by Student's t-test.

Fig. 2: Cyclosporin A delayed mPTP opening and reduced cell death in isolated cardiomyocytes following hypoxia/reoxygenation. 
Cardiomyocytes were co-loaded with calcein- $\mathrm{AM}, \mathrm{CoCl}_{2}$ and propidium iodide (PI). Cyclosporin A (CsA) was introduced 15min before the end of hypoxic period and during the first $10 \mathrm{~min}$ of the reoxygenation phase. Images were acquired every 1-min intervals. Time courses of the changes in calcein and PI signals during reoxygenation in the absence $(A)$ or presence $(B)$ of CsA are shown (signals during equilibration (15min) and hypoxic (2h) phases were omitted for clarity, no significant fluorescence changes being detected). Each trace represents normalized changes of intensities obtained in 9 experiments, each averaging the fluorescence traces of 40-45 cardiomyocytes under the same conditions. Data are presented as percentages $\pm S$.E.M of the maximal initial values for calcein and the final values for PI after Triton addition.

Fig. 3: Two kinetic profiles of mPTP opening during reoxygenation were recorded in cardiomyocytes following hypoxia/reoxygenation.

A: Typical recordings of changes in calcein fluorescence intensity in isolated cardiomyocytes following hypoxia/reoxygenation. The initial fluorescence intensities measured in single cardiomyocytes were normalized to $100 \%$. Each trace represents normalized change in calcein fluorescence during reoxygenation in a single cardiomyocyte (equilibration (15min) and hypoxic $(2 \mathrm{~h})$ periods were omitted for clarity, no significant calcein change being observed). As example, cells 1, 2, 3 and 4 displayed a rapid decrease in calcein fluorescence while cells 5 and 6 showed a slow, gradual decrease. B: the percentage of cells displaying the two modes of mPTP opening at reoxygenation as function of the duration of hypoxia. For each experiment, the response was analyzed by averaging the change in fluorescence obtained from all cardiomyocytes contained in a single 
field (40-45 cardiomyocytes per experiment). Each value is the percentage of cells with opened mPTP $\pm S . E . M$ of 3 to 6 experiments performed with different isolated preparations of cardiomyocytes. ${ }^{*}, \mathrm{P}<0.05$ versus respective values at hypoxia $0.5 \mathrm{~h} ; \dagger, \mathrm{P}<0.05$ versus respective values at hypoxia $1 \mathrm{~h} ; \ddagger, P<0.05$ versus respective values at hypoxia $2 \mathrm{~h}$.

Fig. 4: Effect of antioxidants on reactive oxygen species production during hypoxia/reoxygenation.

Cardiomyocytes were subjected to $2 \mathrm{~h}$ of hypoxia followed by reoxygenation. In some experiments, 2-mercaptopropionylglycine $(400 \mu \mathrm{M})$ and phenanthroline $(10 \mu \mathrm{M})$ were added either throughout the experiment or during hypoxia or $15 \mathrm{~min}$ before the end of hypoxia and during the first $10 \mathrm{~min}$ of reoxygenation. The curve per condition is the average signal \pm S.E.M of 3-6 experiments. Each experiment was calculated by averaging 40-45 traces analyzed from single cardiomyocytes reported as percent of the maximum value achieved during control experiments. A: Cells were co-loaded with calcein-AM and $\mathrm{COCl}_{2}$ and $\mathrm{DHE}$ was added to the equilibration, hypoxia and reoxygenation media. B: Cells were loaded with PI that was also added as well as DCFH-DA to the equilibration, hypoxia and reoxygenation media.

Fig. 5: Time course of mPTP opening and cell death in isolated cardiomyocytes following hypoxia/reoxygenation in the presence or in the absence of antioxidants. Cardiomyocytes were exposed to the protocol of hypoxia/reoxygenation with or without antioxidants added according to 3 different modes as described in MATERIAL and METHODS. Traces during equilibration and hypoxia were omitted 
for clarity, no significant intensities changes being observed. A: Cardiomyocytes were co-loaded with calcein- $\mathrm{AM}$ and $\mathrm{CoCl}_{2}$ and $\mathrm{DHE}$ was added to the equilibration, hypoxia and reoxygenation media. Each trace represents the average change in calcein fluorescence intensity $\pm S$.E.M during reoxygenation obtained from 3-6 experiments. Traces for each experiment were calculated by averaging $40-45$ traces of single cardiomyocytes normalized to $100 \%$. B: PI fluorescence intensity during reoxygenation per condition represents the average intensity \pm S.E.M of 3 to 6 experiments. Each experiment was calculated by averaging 40-45 traces obtained from single cardiomyocytes under the same conditions normalized to $100 \%$ obtained after addition of Triton.

Fig. 6: Effect of cyclosporin A on reactive oxygen species production evaluated by DHE during hypoxia/reoxygenation.

Cardiomyocytes were subjected to $2 \mathrm{~h}$ hypoxia followed by reoxygenation in the presence or in the absence of $1 \mu \mathrm{M}$ cyclosporin $\mathrm{A}$ (CsA) added 15 min before the end of hypoxia and during the first $10 \mathrm{~min}$ of reoxygenation. DHE was added to the equilibration, hypoxia and reoxygenation media. Curve per condition, presented as percent of maximum control value was calculated by averaging 3-6 experiments. Traces per experiment were obtained by evaluating 40-45 single traces of cardiomyocytes contained in a single field. Each point of the curve is the mean \pm S.E.M.

Fig. 7: Effect of cyclosporin A on reactive oxygen species production evaluated by DCFH-DA during hypoxia/reoxygenation. 
Cells were subjected to $2 \mathrm{~h}$ hypoxia followed by reoxygenation in the presence or in the absence of $1 \mu \mathrm{M}$ cyclosporin $\mathrm{A}$ (CsA) added 15 min before the end of hypoxia and during the first 10min of reoxygenation. DCFH-DA was added to the equilibration, hypoxia and reoxygenation media. Curve per condition, presented as percent of maximum control value was calculated by averaging 3-6 experiments. Traces per experiment were obtained by evaluating 40-45 single traces of cardiomyocytes contained in a single field. Each point of the curve is the mean $\pm S . E . M$. 